\title{
THE WHITEHEAD THEOREM FOR NILPOTENT SPACES
}

\section{S. M. GERSTEN}

ABSTRACT. An easy argument is given for the theorem of the title.

E. Dror [1] has published a far-reaching generalization of a classical theorem of J. H. C. Whitehead. An interesting case of Dror's theorem which still causes wonder among topologists is the following result.

Theorem. If $f: X \rightarrow Y$ is a map of connected, pointed, CW complexes which induces an isomorphism on integral homology, and if $X$ and $Y$ are nilpotent spaces, then $f$ is a homotopy equivalence.

We remind the reader that the pointed connected space $X$ is said to be nilpotent if (a) $\pi_{1}(X)$ is a nilpotent group, and (b) for each $n \geq 2$ there is a number $r_{n}>0$ such that $I^{r} \pi_{n}(X)=0$, where $I$ is the augmentation ideal of the group ring $\mathbf{Z}\left[\pi_{1}(X)\right]$.

As in Dror's result, the crucial step is a reduction to a theorem of Stallings.

Lemma 1. The map $f$ of the Theorem induces an isomorphism of fundamental groups.

Proof. Consider the spectral sequences for the fibrations

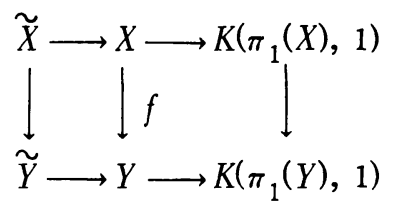

(where $\widetilde{X}, \widetilde{Y}$ are the universal covers of $X, Y$ ). From the low-dimensional terms exact sequences, one deduces that the map $H_{n}\left(\pi_{1}(X, Z) \rightarrow\right.$ $H_{n}\left(\pi_{1}(Y), Z\right)$ is an isomorphis $\mathrm{m}$ for $n=1$ and an epimorphism for $n=2$. Since $\pi_{1}(X)$ and $\pi_{1}(Y)$ are nilpotent groups, Stallings' theorem [2] shows that $\pi_{1}(f): \pi_{1}(X) \rightarrow \pi_{1}(Y)$ is an isomorphism.

Received by the editors November 8, 1973.

AMS (MOS) subject classifications (1970). Primary $18 \mathrm{~F} 25$.

Key words and phrases. Whitehe ad theorem, nilpotent space. 
Continuing with the proof of the Theorem, we may suppose $f: X \hookrightarrow Y$ a cofibration, so $H_{*}(Y, X)=0$. We prove inductively that $\pi_{n}(Y, X)=0$, the induction starting with $n=0$. Let $G=\pi_{1} X=\pi_{1} Y$, the latter identified via $\pi_{1}(f)$. Assume that $\pi_{i}(Y, X)=0$ for $i<n, n>0$. We write the proof in the abelian case $n>1$ in detail (the case $n=1$ requires only a notation change).

Lemma 2. $\pi_{n}(Y, X) / G$-action $=H_{n}(Y, X)$.

This is elementary, proved using the Hurewicz theorem in the universal covering spaces of $X$ and $Y$.

Consider the exact sequence $\pi_{n} Y \stackrel{j}{\rightarrow} \pi_{n}(Y, X) \stackrel{\partial}{\rightarrow} \pi_{n-1}(X)$ and the short exact sequence of $G$-modules $0 \rightarrow \operatorname{Im} j \rightarrow \pi_{n}(Y, X) \rightarrow \operatorname{Im} \partial \rightarrow 0$. Since $\operatorname{Im} j$ and $\operatorname{Im} \partial$ are, respectively, quotient-modules and submodules of $G$-modules, by the nilpotence assumption, there is a number $m>0$ such that

$$
I^{m}(\operatorname{Im} j)=I^{m}(\operatorname{Im} \partial)=0,
$$

where $I$ is the augmentation ideal of $\mathrm{ZG}$. Hence $I^{2 m_{\pi}}{ }_{n}(Y, X)=0$. But by Lemma 2, since $H_{n}(Y, X)=0$, we have $\pi_{n}(Y, X)=I \pi_{n}(Y, X)$. Thus $\pi_{n}(Y, X)$ $=0$.

Thus the relative homotopy groups $\pi_{*}(Y, X)$ are all trivial, and it follows that $f: X \hookrightarrow Y$ is a homotopy equivalence.

\section{REFERENCES}

1. E. Dror, A generalization of the Whitehead theorem, Lecture Notes in Math., vol. 249, Springer-Verlag, Berlin and New York, 1971, pp. 13-22.

2. J. Stallings, Homology and central series of groups, J. Algebra 2 (1965), 170-181. MR 31 \#232.

SCHOOL OF MATHEMATICS, THE INSTITUTE FOR ADVANCED STUDY, PRINCETON, NEW JERSEY 08540

Current address: Department of Mathematics, University of Illinois, Urbana, Illinois 61801 